\title{
Experimental osteonecrosis: development of a model in rodents administered alendronate
}

\section{Nicolau CONTE NETO(a) Luis Carlos SPOLIDORIO(b) Cleverton Roberto de ANDRADE ${ }^{(b)}$ Jônatas Caldeira ESTEVES(c) Elcio MARCANTONIO JR. (d) $^{\text {(d) }}$}

(a) Centro Universitário do Estado do Pará - CESUPA, School of Dentistry, Department of Oral Surgery, Belem, PA, Brazil

(b) Universidade Estadual Paulista - UNESP, School of Dentistry, Department of Physiology and Pathology, Araraquara, SP, Brazil

(c) Universidade Federal do Rio de Janeiro - UFRJ, School of Dentistry, Department of Dental Clinics, Rio de Janeiro, RJ, Brazil

(d) Universidade Estadual Paulista - UNESP, School of Dentistry, Department of Diagnosis and Surgery, Araraquara, SP, Brazil

Declaration of Interests: The authors certify that they have no commercial or associative interest that represents a conflict of interest in connection with the manuscript.

\section{Corresponding Author:}

Nicolau Conte Neto

E-mail: conteneto@hotmail.com

DOI: 10.1590/1807-3107BOR-2016.vol30.0099

Submitted: December 17, 2015

Accepted for publication: May 23,2016

Last revision: June 09, 2016

\begin{abstract}
The main objective of this study was to cause bisphosphonate-related osteonecrosis of the jaws to develop in a rodent model. Adult male Holtzman rats were assigned to one of two experimental groups to receive alendronate (AL; $1 \mathrm{mg} / \mathrm{kg} /$ week; $\mathrm{n}=6$ ) or saline solution (CTL; $\mathrm{n}=6$ ). After 60 days of drug therapy, all animals were subjected to first lower molar extraction, and 28 days later, animals were euthanized. All rats treated with alendronate developed osteonecrosis, presenting as ulcers and necrotic bone, associated with a significant infection process, especially at the inter-alveolar septum area and crestal regions. The degree of vascularization, the levels of C-telopeptide cross-linked collagen type I and bone-specific alkaline phosphatase, as well as the bone volume were significantly reduced in these animals. Furthermore, on radiographic analysis, animals treated with alendronate presented evident sclerosis of the lamina dura of the lower first molar alveolar socket associated with decreased radiographic density in this area. These findings indicate that the protocol developed in the present study opens new perspectives and could be a good starting model for future property design.
\end{abstract}

Keywords: Osteonecrosis; Alendronate; Infection.

\section{Introduction}

In recent years, public health statistics have included the bisphosphonates (Bps) among the most common drugs prescribed in the world, since they are highly effective in the treatment of several bone diseases. ${ }^{1}$ However, since 2003, concerns have been raised regarding the increasing side-effects of Bps.

In this context, Bps-related osteonecrosis of the jaws (BRONJ) represents a challenge in science. Even with the numerous efforts to develop experimental models of this disease, none of the BRONJ pathogenesis hypotheses has yet been completely accepted. Conversely, strong correlations have been made between this bone necrosis and possible co-factors, including Bps type and treatment length, ${ }^{2}$ as well as trigger agents, highlighting surgical procedures with bone manipulation, such as dental implants and tooth extractions. ${ }^{3}$

Analysis of data retrieved from clinical studies indicates a strong association between tooth extractions and BRONJ., ${ }^{4,5}$ Thus, the effects of Bps therapy on the healing of alveolar sockets have been the focus of several in vivo studies. ${ }^{6,7}, 8$ Recently, we demonstrated the development of BRONJ-like lesions in rodents by associating tooth extractions with daily high dosages of alendronate (ALN) in a long-term study. ${ }^{9}$ However, 
studies using lower dosages of Bps showed only a transient impairment of alveolar socket healing after tooth extractions. ${ }^{10,11}$

Within this context, the aim of this study was to develop BRONJ lesions in rodents by means of lower doses of ALN, which, in rats, corresponded with the cumulative dosages for the management of rheumatic diseases. ${ }^{12}$ Furthermore, we undertook a critical discussion of the reasons behind the strong association between tooth extractions and this disease.

\section{Methodology}

\section{Animals and reagents}

Twelve male adult Holtzman rats (Rattus norvergicus albinus), weighing approximately $200 \mathrm{~g}$ each, were housed under similar conditions in cages with access to food and water ad libitum. All experimental protocols were approved by the institution's Ethics Committee for the Use of Experimental Animals (no. 18/2009) and performed in accordance with the guidelines of the Brazilian National Council for the Control of Animal Experimentation.

Alendronate (ALN) was purchased from ALCON Laboratory (São Paulo, SP, Brazil). The drug was dissolved in sterile physiological saline $(0.9 \% \mathrm{NaCl})$ and diluted to the specified concentration.

\section{Experimental design}

After a 3-day acclimatization period, 6 rats were treated with weekly doses of alendronate $\left(1 \mathrm{mg} / \mathrm{kg}\right.$ body weight), ${ }^{12}$ and 6 rats were treated with weekly doses of vehicle alone ( $0.9 \%$ saline), both subcutaneously.

After 60 days of the pharmacologic therapy, all animals were subjected to left lower first molar (M1) extraction under general anesthesia by a combination of ketamine chlorhydrate (Ketamina Agener, Agener União Ltda, São Paulo, SP, Brazil; 0.08 mL/100 g body weight) and $2 \%$ xylazine (Rompum, Bayer S.A., São Paulo, SP, Brazil; 0.04 mL/100 g body weight).

The same professional performed the tooth extractions using the same technique in all animals. Initially, the rats were placed in a dorsal position and fixed in a special device. The surrounding gingivae were carefully detached from the lower first molars with a dental explorer. Then, with a Hollenback carver, the tooth was luxated and separated into 2 segments that were removed with a forceps adapted around the cervical lines of the segments.

After the surgical procedure, all animals received an intramuscular dose of antibiotic (Pentabiótico ${ }^{\circledR}$, Wyeth-Whitehall Ltda, São Paulo, Brazil, 0.1 mg/Kg) and anti-inflammatory Ketoflex (Ketoprofen 1.0\%, MERIAL, São Paulo, Brazil, 0.03 mL/rat). Animals were euthanized by anesthesia overdose at 28 days after tooth extractions, and the ALN or sterile physiological saline administration protocol was maintained until the animals' death.

\section{Specimen processing}

All tissue blocks were immersed directly in $10 \%$ buffered formalin fixative solution for $48 \mathrm{~h}$. After that, 6 specimens of each group/period were subjected to routine histological processing for descriptive and stereometric evaluation. All specimens were decalcified in tetrasodium-EDTA aqueous solution $(0.5 \mathrm{M}, \mathrm{pH} 7.4)$ for 2-3 months, under agitation at room temperature. All specimens were then processed and included in paraffin blocks. Serial $4-\mu \mathrm{m}$ sections were obtained in the bucco-lingual direction, stained with hematoxylin and eosin, and referred for evaluation by light microscopy (Leica DM1200M; Leica Microsystems, Wetzlar Hesse, Germany).

\section{Histological and stereometric analyses}

One Board-certified oral pathologist blinded to the group assignments performed these analyses at three distinct times to minimize discrepancy in the scores (kappa index $=0.76$ ). The histological endpoints were evaluated at 4 fields in each section (cervical and apical thirds of the mesial and distal sockets) at magnifications of 100x, 200x, 400x, and 1000x. Evaluation included the degrees of bone necrosis (areas of empty osteocytic lacunae and heavily eroded surfaces) and infection (bacteria proportion and extension of biofilm organization). The inflammation was evaluated according to quantity and quality based on the density, type, and distribution of inflammatory cell infiltrate. ${ }^{6}$ Qualitatively, the inflammation was classified as acute (predominance of neutrophil infiltration), chronic (predominance of mononuclear 
cell infiltration, such as macrophages and lymphocytes), and mixed (presence of cells of both acute and chronic processes in similar proportions). Furthermore, the degree of vascularization (number of vessels) was also evaluated. These parameters were scored on a four-point scale: 0 (absent; 0\%), 1 (mild; $\leq 10 \%$ ), 2 (moderate; $>10$ and $\leq 50 \%$ ), and 3 (increased; $>50 \%$ ).

Stereometric analysis was performed with Leica Application software Suite 3.8.0 (Leica Microsystems LTD, Heerbrugg, Switzerland). The measurements were performed at the distal root of the left M1 at the regions of interest (ROIs) that included 3 different areas inside the socket. Initially, a standard ROI was determined by identification of a quadrangular area extending from the level of the cemento-enamel junction (CEJ) of the left second mandible (M2) to the apical end of the M2 and between the mesial and distal alveolar bone surfaces. This quadrangular area was divided into 3 equal ROIs $(1,2,3){ }^{9}$

The variables analyzed included the percentage of the root socket filled with bone tissue (BV) in each ROI, following the nomenclature and abbreviations recommended by the American Society for Bone and Mineral Research. ${ }^{13}$ Bone volume (\%) represented bone volume $\left(\mathrm{mm}^{3}\right)$ per total tissue volume $\left(\mathrm{mm}^{3}\right)$. All analyses were performed at a magnification of 100x, and 3 measurements were taken for each specimen, to complete the stereometric analysis. The distance between the selected sections was $50 \mu \mathrm{m}$.

\section{Image acquisition}

For radiographic evaluation, a digital radiograph of the left alveolar socket was taken after euthanasia. The left mandible was fixed in a holding device with the vertical long axis of alveolar socket perpendicular to the central $x$-ray beam and parallel to the sensor at a $40-\mathrm{cm}$ focus-object distance. The $\mathrm{x}$-ray unit was operated at $70 \mathrm{KVp}, 10 \mathrm{~mA}$, and $0.2 \mathrm{~s}$ (Expectro 70x, Dabi Atlante, Ribeirão Preto, SP, Brazil).

\section{Radiographic bone density}

A single blinded calibrated examiner evaluated mandibular radiographs. The radiographic bone density in the mandible was determined by analysis of the gray scale in an area of $15 \times 15$ pixels at the ROIs, including 3 different regions within the distal root of the left M1, as described previously. Furthermore, we also evaluated one region immediately after the mesial socket of left M1, which corresponded to the body's mandibular bone density (ROI 4).

This analysis was done with the image-analysis software Image Tool 2.03 (UTHSCA, San Antonio, Texas, USA), which provided the gray scale average and standard deviation in these pre-determined regions by means of a histograph. The bone density calculations were performed based on the average gray levels of the evaluated ROIs, which were divided by the gray level of the implant, to compensate for minimal differences among radiographs, since the density of the metallic standard was the same in all specimens. ${ }^{14}$

\section{Assessment of bone turnover biochemical markers}

Blood samples were collected on the day of euthanasia by cardiac puncture and centrifuged for plasma separation. The levels of serum collagen type 1 cross-linked C-telopeptide (CTX) and bone-specific alkaline phosphatase (BALP) were determined by enzyme-linked immunosorbent assay kits (CUSABIO BIOTECH CO., Ltd, Wuhan, P.R. China). ${ }^{9}$

\section{Statistical analysis}

Data were initially subjected to the Kolmogorov-Smirnov normality test. We then performed comparisons among groups and periods for non-parametric data, using the Kruskal-Wallis test, followed by Dunn's multiple-comparison test or analysis of variance (ANOVA), then Tukey tests for parametric data. The data were evaluated by means of GraphPad Prism 5.0 (San Diego, CA, USA), and statistical significance was set at $p<0.05$ with 95\% confidence intervals.

\section{Results}

\section{Clinical and histological features}

All animals of the AL group exhibited different degrees of bone exposure (Figure 1a), while control animals presented with complete epithelial healing without signs of inflammation (Figure 1b).

A marked bone necrosis associated with an infection process was observed in animals treated 
with ALN, especially at the inter-alveolar septum area (Figure 2b), while control animals displayed none of these features $(p<0.0001)$ (Figures $2 a, 3 a, 3 b)$. Furthermore, animals in the AL group presented a lower degree of vascularization $(p<0.01)$ when compared with those in the control group (Figure 4a), while no statistically significant differences were found concerning the inflammation quality and degree between these groups of animals (Figures 3c, 3d).

Regarding BV count, we observed that, at ROI 1 and ROI 2, animals of the AL group presented rates significantly less than those of CTL animals $(p<0.01)$, while no statistically significant differences were found in ROI 3 (Figure 4b).
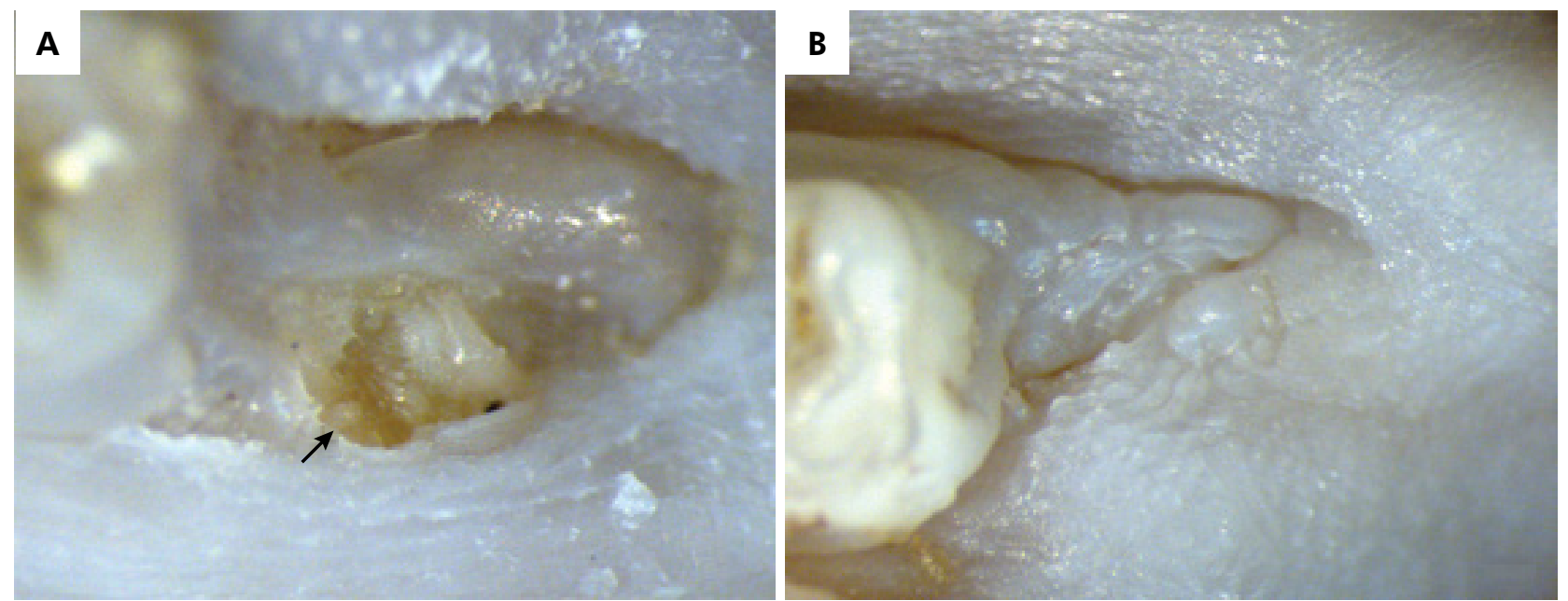

Figure 1. Clinical aspects of a lower first molar (M1) alveolar socket 28 days after tooth extraction. Animals treated with ALN (A) exhibited areas of exposed bone with a marked impairment of alveolar socket re-epithelialization. In contrast, animals treated with sterile physiological saline (B) presented a full epithelial lining on the alveolar socket, with no signs of inflammation or infection (asterisk).


Figure 2. Histologic sections of tooth sockets 28 days after left lower first molar extraction and stained with hematoxylin and eosin. The animals in CTL group (A) demonstrated alveolar socket area completely remodeled, with no signs of bone necrosis (asterisk), while in the ALN group (B) it was observed the inter-radicular septum retention, which was associated with bone necrosis (asterisk) and infection (arrow; original magnification $\times 100$ ). 
A
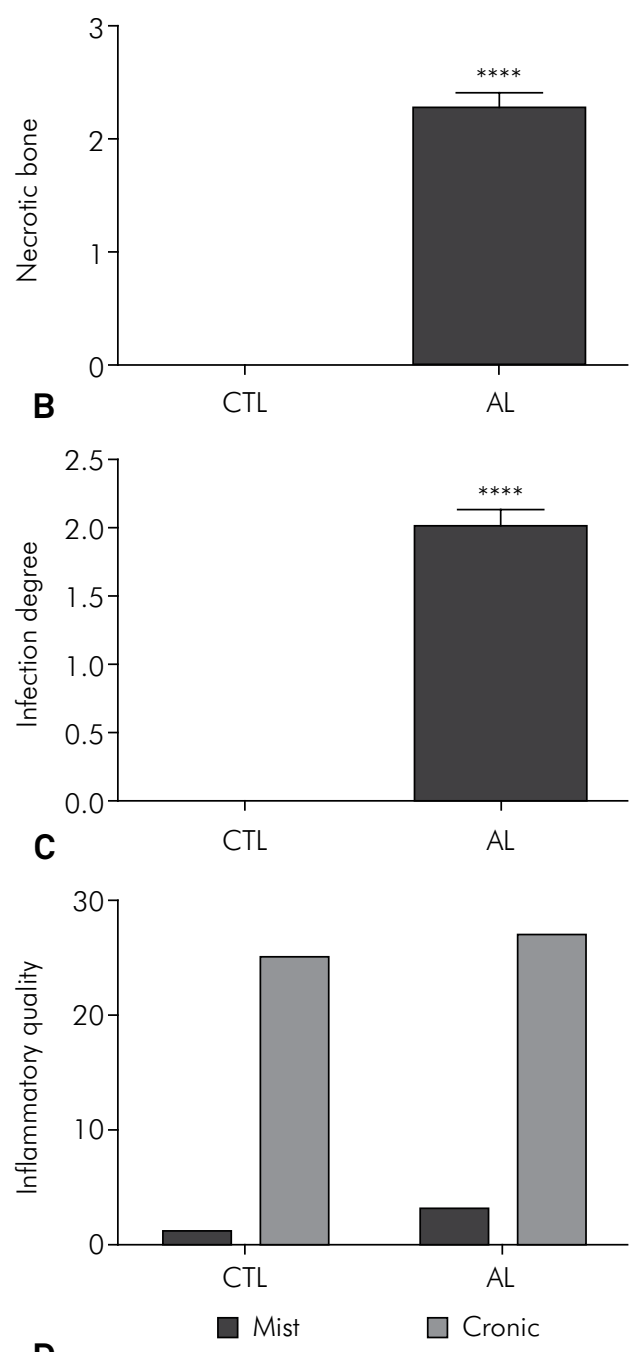

D

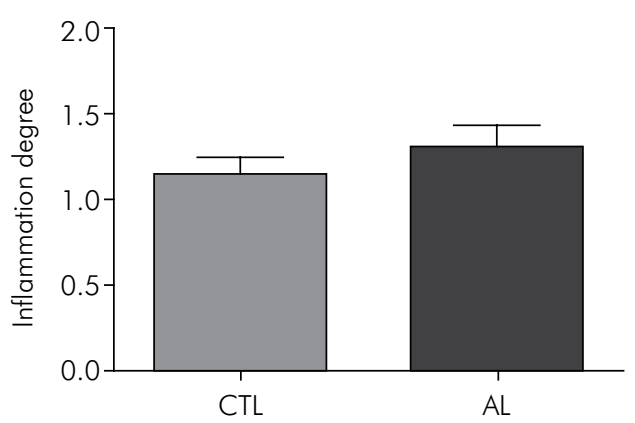

Figure 3. The effects of ALN therapy on bone necrotic levels (A), degrees of infection (B), and on the quality (C) and degree (D) of inflammation at 28 days after tooth extraction. (A) Animals treated with ALN developed a higher proportion of bone necrosis compared with matched controls $\left({ }^{* * *} p<0.0001\right)$. (b) Animals treated with ALN presented an increased degree of infection compared with matched controls $\left({ }^{* * * *} p<0.0001\right)$. No differences were found regarding the quality (C) and degree (D) of inflammation between the groups studied.

\section{A}
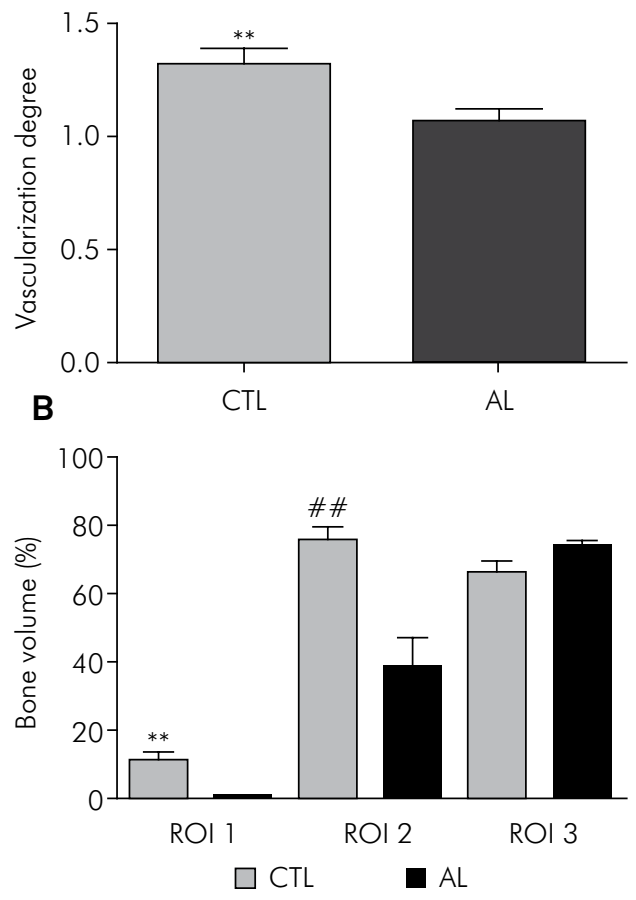

Figure 4. The effects of ALN therapy on the degree of vascularization and bone volume (\%) in experimental groups 28 days after tooth extractions. (A) Animals of the CTL group developed a higher degree of vascularization compared with those treated with ALN (**p < 0.01). (B) Regions of Interest (ROI) 1 and 2 of CTL animals exhibited a statistically significant increase in bone volume compared with ROIs 1 and 2 of animals treated with ALN (** $^{* *}<0.01$ in relation to ROI 1 of $\mathrm{AL} ; \# \# \mathrm{p}<0.01$ in relation to $\mathrm{ROI} 2$ of $\mathrm{AL}$ ).

\section{Radiographic aspects}

In the radiographic analysis, it could be observed that animals treated with ALN showed a degree of bone radiographic density $(\mathrm{BRD})$ significantly less at ROI $1(\mathrm{p}<0.01)$ and ROI $2(\mathrm{p}<0.001)$ when compared with that of control animals (Figure 5). Furthermore, in the AL group, the linings of the alveolar socket were more evident when compared with those of control animals and also presented sclerosis on the M1 alveolar lamina dura (Figures 6a, 6b), as well as the highest values of BRD at the ROI 4 area $(\mathrm{p}<0.001)$ (Figure 5).

\section{Bone metabolism markers}

Animals treated with ALN presented the lowest values of BALP $(p<0.01)$ and CTX $(p<0.001)$ when compared with control animals (Figures $7 \mathrm{a}, 7 \mathrm{~b}$ ). 


\section{Discussion}

Given the large number of patients developing BRONJ worldwide, several animal models of BRONJ-like lesions have been recently published in an effort to further explore the disease's pathophysiology. However, there is no standard BRONJ experimental model, since most models fail to reproduce clinical reality and do not associate several co-factors that

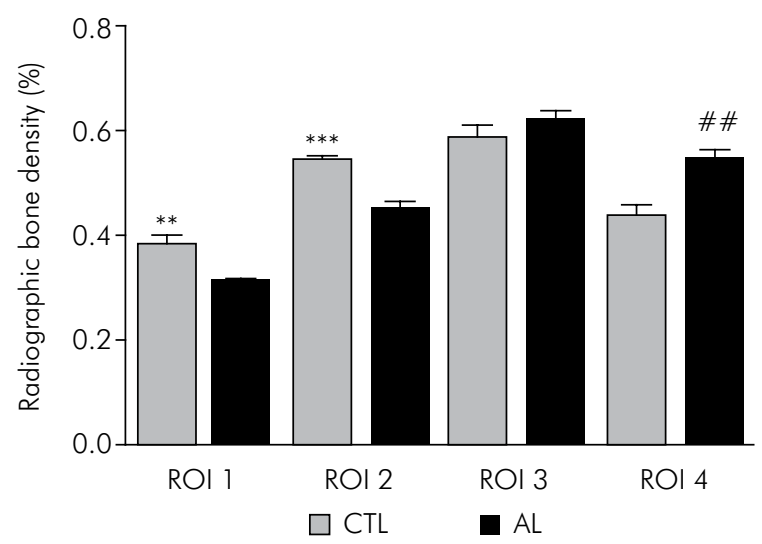

Figure 5. Percentage of radiographic bone density (\%) in experimental groups 28 days after tooth extractions. ROls 1 and 2 of CTL animals exhibited a statistically significant increase in radiographic bone density compared with ROls 1 and 2 of animals treated with $\mathrm{ALN}\left({ }^{* *} \mathrm{p}<0.01\right.$ in relation to $\mathrm{ROI} 1$ of $\mathrm{AL}$; ${ }^{* * *} \mathrm{p}<0.001$ in relation to $\mathrm{ROI} 2$ of $\mathrm{AL}$ ). Furthermore, $\mathrm{ROI} 4$ of ALN-treated animals exhibited a statistically significant increase in radiographic bone density compared with $\mathrm{ROI} 4$ of matched controls ( $\# \# p<0.01$ in relation to $R O I$ of $A L)$. undertake a reliable cause-effect relationship between Bps and disease pathogenesis. $6,7,8,9,15,16,17,18,19,20,21,22,23,24$ To the best of our knowledge, this is the first study to demonstrate $100 \%$ of BRONJ-like lesions by associating tooth extractions with alendronate in cumulative dosages for the management of rheumatic diseases. ${ }^{12}$

The first advantage of our experimental model is the high percentage of bone necrosis achieved. The rate of bone lesions in previously published experimental models varies from 0 to $100 \%$, $6,7,8,9,15,16,17,18,19,20,21,22,23,24$ and only four papers have reported $100 \%$ of BRONJ-like lesions ${ }^{89,17,18}$ However, models that reported a bone lesion rate of $100 \%$ were developed by exposing animals to supra-therapeutic dose regimens ${ }^{9,17}$ or to a high-intensity trauma, ${ }^{8,18}$ which represents an artificial scenario. In contrast, we proposed an experimental model that not only achieved $100 \%$ of BRONJ-like lesions in rodents, but that also resembled human disease from clinical, radiological, and histological aspects. Additionally, our model simulated a more clinical reality of benign diseases than previous models, since we associated a less aggressive bisphosphonate schedule (type and dose) with minimal surgical trauma.

In our experimental model, several aspects had to be considered, specifically regarding the Bps regimen, which seems to be a key point in the disease's development. Our study is the first to reproduce BRONJ-like lesions using ALN alone without the association of other

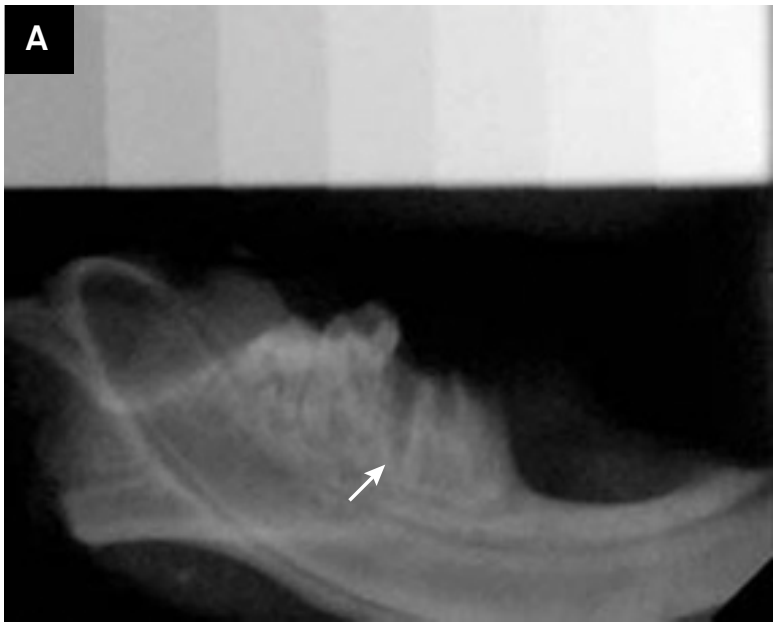

\section{B}

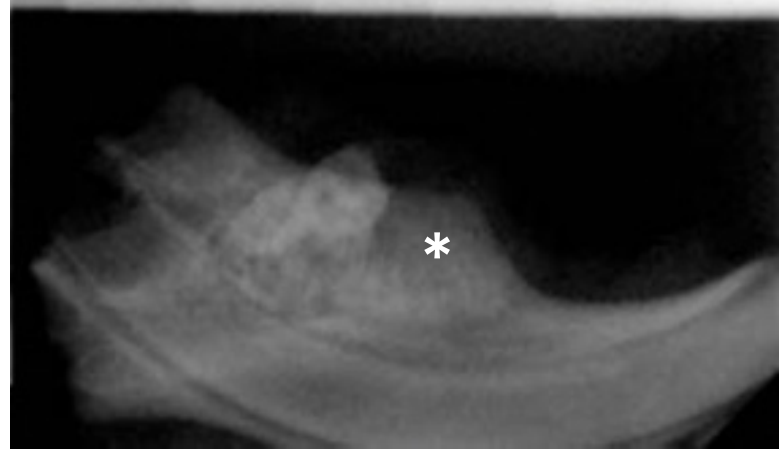

Figure 6. Radiographic features of the alveolar socket healing after 28 days of tooth extraction in animals treated with ALN (A) and sterile physiological saline (B). In the AL group (a), the linings of the alveolar socket (arrow) are more evident compared with those of the control animals (asterisk) and also presented sclerosis on the M1 alveolar lamina dura. 
A

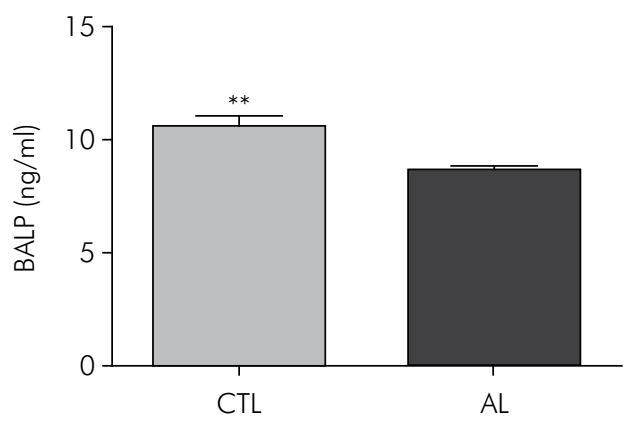

B

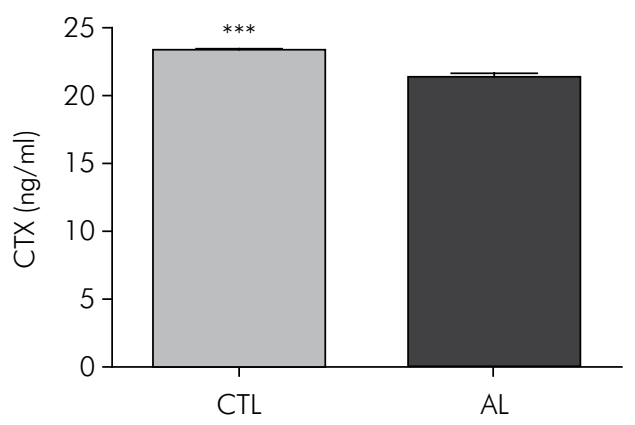

Figure 7. Levels of BALP (A) and CTX (B) in experimental groups at 28 days after tooth extractions. In the CTX analysis, animals in the CTL group presented levels statistically significantly higher than those of animals in the $\mathrm{AL}$ group ( $\left.{ }^{* * *} \mathrm{p}<0.001\right)$. In the BALP analysis, CTL animals exhibited increased levels of BALP compared with those in the AL group (**p $<0.01)$.

drugs or comorbidities. While some studies that used alendronate did not demonstrate BRONJ-like lesions, ${ }^{10,11}$ others did not adopt supra-therapeutic dose regimens, as aforementioned, ${ }^{9}$ or steroids. ${ }^{20,24}$ Furthermore, most experimental models reproduced oncologic schedules with high dosages of zolendronate. ${ }^{78,15,16,18,21,22,23,25}$ However, although zolendronate is undeniably important, it is essential to note that ALN is among the most common Bps prescribed worldwide and is specifically indicated for the treatment of benign diseases, such as osteoporosis, osteopenia, and rheumatic diseases. ${ }^{1}$

In this context, to compensate for the differences in potency between alendronate and zolendronate, as well as for the lower plasma concentrations when the subcutaneous route rather than the intravenous route is used, ${ }^{26}$ we conclude that the total Bps dose administered over a long period of time is important for the magnitude of the reduction in bone turnover. ${ }^{27}$ Indeed, animals in the AL group presented the lowest levels of CTX and BALP, which are markers of bone metabolism, ${ }^{28}$ thereby supporting the effectiveness of our Bps treatment schedule in suppressing bone turnover at a key point in disease development.

In the scenario of clinical reality, some studies selected experimental animals with systemic or metabolic diseases to simulate the physiologic disparity between these animals and healthy animals. ${ }^{7,15,16,20,26}$ We do not rule out the relevance of these studies, but rather believe that healthy animals provide some relevant advantages. First, the more variables included in the experimental model, the more difficult it is to determine the true role of Bps in disease pathogenesis. Second, Bps have several applications even in healthy patients, including therapeutic options for improving the bone formation process and reducing resorption in different bone-grafting procedures, ${ }^{29}$ implant surface treatments, ${ }^{30}$ and as antibiofilm agents. ${ }^{31}$

Although some authors have demonstrated spontaneous BRONJ lesions, ${ }^{9}$ a local trigger factor seems highly relevant to the induction of this disease in animal models. The main local agents include periodontal disease, ${ }^{15,23}$ bacterial injections in addition to bone defects, ${ }^{22}$ and tooth extractions. ${ }^{916,17,18,19,20,21,24,25,26}$ The last is undoubtedly the most relevant, since most patients develop BRONJ lesions after tooth extractions, as described in clinical studies. ${ }^{4,5}$ This finding could be related to an urgent requirement for wound healing after this surgical procedure, especially during initial phases, and, in the field of Bps, this is worsened since these drugs impair the angiogenesis and resorption process after tooth extractions. ${ }^{11,32}$ Indeed, these features are in agreement with our findings of lower CTX levels and degrees of vascularization in animals treated with ALN. Therefore, the association of a high demand requirement in an already compromised bone-remodeling environment could result in the collapse of the repaired wound.

In contrast, some authors believe that $\mathrm{BRONJ}$ may be associated with changes in oral bacterial behaviors due to increased bacterial activity. 22,32 Indeed, as in this study, other studies have demonstrated areas of active infection ${ }^{26}$ and necrotic bone at the inter-alveolar bone region that have been attributed to the prolonged retention of this area. ${ }^{10,11}$ 
In this discussion, it is important to state that even in the presence of a relevant infection process in animals treated with ALN, no differences in the inflammatory responses were found between the groups. This is particularly interesting since a microbial infection typically triggers host immune responses ${ }^{33}$ and therefore enhances the degree of inflammation. A possible explanation for this finding is the dose-dependent anti-inflammatory effect of $\mathrm{Bps}^{34}$ that could, over the long term, lead to immunosuppressive conditions favoring the development of infections.

Our research into infection issues also demonstrated various degrees of impairment in soft tissue coverage in animals treated with ALN, which is relevant due to the essential role of epithelial coverage protection from oral bacterial infection. ${ }^{24}$ Indeed, it has been demonstrated that experimental BRONJ lesions in rodents can be prevented by immediate mucoperiosteal coverage. ${ }^{24}$ This could be of concern, since Bps inhibits the migration of oral epithelial cells, ${ }^{32}$ and, after tooth extraction, there is a high demand for such migration, considering that the degree of reepithelialization depends on the depth and width of the wound. ${ }^{35}$

One of the BRONJ diagnostic criteria is the persistence of bone exposure in the oral cavity for at least 8 weeks. ${ }^{2}$ In our opinion, this concept cannot be strictly extended to animal experimental models, due to inherent metabolic differences between these species. Although both rodents and humans share similar sequences of alveolar healing, it occurs much more rapidly in rats than in humans. ${ }^{36}$ Furthermore,

\section{References}

1. Rogers MJ, Gordon S, Benford HL, Coxon FP,

Luckman SP, Monkkonen J et al. Cellular and molecular mechanisms of action of bisphosphonates. Cancer. 2000;88(12 Suppl):2961-78. doi:10.1002/10970142(20000615)88:12+<2961::AID-CNCR12>3.0.CO;2-L

2. Ruggiero SL, Dodson TB, Assael LA, Landesberg R, Marx RE, Mehrotra B. American Association of Oral and Maxillofacial Surgeons position paper on bisphosphonate-related osteonecrosis of the jaws - 2009 update. J Oral Maxillofac Surg. 2009;67(5 Suppl):2-12. doi:10.1016/j.joms.2009.01.009 when transient, the negative effects of Bps on socket healing have not been extended beyond 14 days. ${ }^{10,11}$

In this study, we found lower BV values at the medial and apical regions in the AL group, compatible with the lowest levels of BALP in this group. These features are in agreement with those reported by authors who found inhibitory effects of ALN on osteogenesis. ${ }^{32}$ In contrast, we also observed no statistically significant differences in bone volume at the apical socket area between the groups, which reinforces, at least in part, the theories of other authors who found no direct effects of Bps on the ability of osteoblasts to produce bone matrix in vivo. ${ }^{37}$

\section{Conclusion}

Within the limitations of this study, our results clearly demonstrated that the association of ALN therapy and tooth extraction was able to induce BRONJ-like lesions in rodents. This study provides an experimental model that achieved $100 \%$ of jaw lesions resembling human disease in clinical, radiological, and histological aspects, which could be useful in the development of preventive and therapeutic approaches to the treatment of this disease, as well as to further studies addressing other issues related to BRONJ pathways, including other trigger agents.

\section{Acknowledgments}

This study was supported by the State of São Paulo Research Foundation and the National Council for Scientific and Technological Development.

3. Marx RE, Cillo JE Jr, Ulloa JJ. Oral

bisphosphonate-induced osteonecrosis: risk factors, prediction of risk using serum CTX testing, prevention, and treatment. J Oral Maxillofac Surg. 2007;65(12):2397-410. doi:10.1016/j.joms.2007.08.003

4. Thumbigere-Math V, Sabino MC, Gopalakrishnan R, Huckabay S, Dudek AZ, Basu S et al. Bisphosphonate-related osteonecrosis of the jaw: clinical features, risk factors, management, and treatment outcomes of 26 patients. J Oral Maxillofac Surg. 2009;67(9):1904-13. doi:10.1016/j.joms.2009.04.051 
5. Vieillard MH, Maes JM, Penel G, Facon T, Magro L, Bonneterre J et al. Thirteen cases of jaw osteonecrosis in patients on bisphosphonate therapy. Joint Bone Spine. 2008;75(1):34-40. doi:10.1016/j.jbspin.2007.05.003

6. Sonis ST, Watkins BA, Lyng GD, Lerman MA, Anderson $\mathrm{KC}$. Bony changes in the jaws of rats treated with zoledronic acid and dexamethasone before dental extractions mimic bisphosphonate-related osteonecrosis in cancer patients. Oral Oncol. 2009;45(2):164-72. doi:10.1016/j.oraloncology.2008.04.013

7. Hokugo A, Christensen R, Chung EM, Sung EC, Felsenfeld AL, Sayre JW et al. Increased prevalence of bisphosphonate-related osteonecrosis of the jaw with vitamin D deficiency in rats. J Bone Miner Res. 2010;25(6):1337-49. doi:10.1002/jbmr.23

8. Biasotto M, Chiandussi S, Zacchigna S, Moimas S, Dore F, Pozzato G et al. A novel animal model to study non-spontaneous bisphosphonates osteonecrosis of jaw. J Oral Pathol Med. 2010;39(5):390-6. doi:10.1111/j.1600-0714.2009.00878.x

9. Conte Neto N, Spolidorio LC, Andrade CR, Bastos AS, Guimarães M, Marcantonio E Jr. Experimental development of bisphosphonate-related osteonecrosis of the jaws in rodents. Int J Exp Pathol. 2013;94(1):65-73. doi:10.1111/iep.12007

10. Hikita H, Miyazawa K, Tabuchi M, Kimura M, Goto S. Bisphosphonate administration prior to tooth extraction delays initial healing of the extraction socket in rats. J Bone Miner Metab. 2009;27(6):663-72. doi:10.1007/s00774-009-0090-6

11. Aguirre JI, Altman MK, Vanegas SM, Franz SE, Bassit AC, Wronski TJ. Effects of alendronate on bone healing after tooth extraction in rats. Oral Dis. 2010;16(7):674-85. doi:10.1111/j.1601-0825.2010.01677.x

12. Kawabata A, Kawao N, Hironaka Y, Ishiki T, Matsunami M, Sekiguchi F. Antiallodynic effect of etidronate, a bisphosphonate, in rats with adjuvant-induced arthritis: involvement of ATP-sensitive K+ channels. Neuropharmacology. 2006;51(2):182-90. doi:10.1016/j.neuropharm.2006.03.015

13. Parfitt AM. Bone histomorphometry: standardization of nomenclature, symbols and units (summary of proposed system). Bone. 1988;9(1):67-9. doi:10.1016/8756-3282(88)90029-4

14. Sakakura CE, Margonar R, Sartori R, Morais JA, Marcantonio E Jr. The influence of cyclosporin a on mechanical retention of dental implants previously integrated to the bone: a study in rabbits. J Periodontol. 2006;77(12):2059-62. doi:10.1902/jop.2006.050290

15. Li CL, Lu WW, Seneviratne CJ, Leung WK, Zwahlén RA, Zheng LW. Role of periodontal disease in bisphosphonate-related osteonecrosis of the jaws in ovariectomized rats. Clin Oral Implants Res. 2016;27(1):1-6. doi:10.1111/clr.12502

16. Kim JW, Landayan ME, Lee JY, Tatad JC, Kim SJ, Kim MR et al. Role of microcracks in the pathogenesis of bisphosphonate-related osteonecrosis of the jaw. Clin Oral Investig. 2016 [Epub ahead of print]. doi:10.1007/s00784-016-1718-2
17. Jang HW, Kim JW, Cha IH. Development of animal model for Bisphosphonates-related osteonecrosis of the jaw (BRONJ). Maxillofac Plast Reconstr Surg. 2015;37(1):18. doi:10.1186/s40902-015-0020-6

18. Howie RN, Borke JL, Kurago Z, Daoudi A, Cray J, Zakhary IE et al. A model for osteonecrosis of the jaw with zoledronate treatment following repeated major trauma. PLoS One. 2015;10(7):e0132520. doi:10.1371/journal.pone.0132520

19. Ersan N, van Ruijven LJ, Bronckers AL, Olgaç V, Ilgüy D, Everts V. Teriparatide and the treatment of bisphosphonate-related osteonecrosis of the jaw: a rat model. Dentomaxillofac Radiol. 2014;43(1):20130144. doi:10.1259/dmfr.20130144

20. Berti-Couto SA, Vasconcelos AC, Iglesias JE, Figueiredo MA, Salum FG, Cherubini K. Diabetes mellitus and corticotherapy as risk factors for alendronate-related osteonecrosis of the jaws: a study in Wistar rats. Head Neck. 2014;36(1):84-93. doi:10.1002/hed.23260

21. Barba-Recreo P, Del Castillo Pardo de Vera JL, García-Arranz M, Yébenes L, Burgueño M. Zoledronic acid - related osteonecrosis of the jaws. Experimental model with dental extractions in rats. J Craniomaxillofac Surg. 2014;42(6):744-50. doi:10.1016/j.jcms.2013.11.005

22. Tsurushima H, Kokuryo S, Sakaguchi O, Tanaka J, Tominaga K. Bacterial promotion of bisphosphonate-induced osteonecrosis in Wistar rats. Int J Oral Maxillofac Surg. 2013;42(11):1481-7. doi:10.1016/j.ijom.2013.06.011

23. Kang B, Cheong S, Chaichanasakul T, Bezouglaia O, Atti E, Dry SM et al. Periapical disease and bisphosphonates induce osteonecrosis of the jaws in mice. J Bone Miner Res. 2013;28(7):1631-40. doi:10.1002/jbmr.1894

24. Abtahi J, Agholme F, Aspenberg P. Prevention of osteonecrosis of the jaw by mucoperiosteal coverage in a rat model. Int J Oral Maxillofac Surg. 2013;42(5):632-6. doi:10.1016/j.ijom.2013.02.007

25. Yang H, Pan H, Yu F, Chen K, Shang G, Xu Y. A novel model of bisphosphonate-related osteonecrosis of the jaw in rats. Int J Clin Exp Pathol. 2015;8(5):5161-7.

26. Kim JW, Tatad JC, Landayan ME, Kim SJ, Kim MR. Animal model for medication-related osteonecrosis of the jaw with precedent metabolic bone disease. Bone. 2015;81:442-8. doi:10.1016/j.bone.2015.08.012

27. Chapurlat RD, Delmas PD. Drug insight: bisphosphonates for postmenopausal osteoporosis. Nat Clin Pract Endocrinol Metab. 2006;2(4):211-9. doi:10.1038/ncpendmet0121

28. Pagani F, Francucci CM, Moro L. Markers of bone turnover: biochemical and clinical perspectives. J Endocrinol Invest. 2005;28(Suppl 10):8-13.

29. Ayranci F, Gungormus M, Omezli MM, Gundogdu B. The effect of alendronate on various graft materials used in maxillary sinus augmentation: a rabbit study. Iran Red Crescent Med J. 2015;17(12):e33569. doi:10.5812/ircmj.33569 
30. Boanini E, Torricelli P, Forte L, Pagani S, Mihailescu N, Ristoscu C et al. Antiresorption implant coatings based on calcium alendronate and octacalcium phosphate deposited by matrix assisted pulsed laser evaporation. Colloids Surf B Biointerfaces. 2015;136:449-56. doi:10.1016/j.colsurfb.2015.09.044

31. Reshamwala SM, Mamidipally C, Pissurlenkar RR, Coutinho EC, Noronha SB. Evaluation of risedronate as an antibiofilm agent. J Med Microbiol. 2016;65(1):9-18. doi:10.1099/jmm.0.000193

32. Kobayashi Y, Hiraga T, Ueda A, Wang L, Matsumoto-Nakano M, Hata K et al. Zoledronic acid delays wound healing of the tooth extraction socket, inhibits oral epithelial cell migration, and promotes proliferation and adhesion to hydroxyapatite of oral bacteria, without causing osteonecrosis of the jaw, in mice. J Bone Miner Metab. 2010;28(2):165-75. doi:10.1007/s00774-009-0128-9

33. Schaudinn C, Gorur A, Keller D, Sedghizadeh PP, Costerton JW. Periodontitis: an archetypical biofilm disease. J Am Dent Assoc. 2009;140(8):978-86. doi:10.14219/jada.archive.2009.0307

34. Dunn CJ, Slatter JG, Nugent RA. Bisphosphonates: a review of their novel anti-inflammatory properties and potential for the treatment of rheumatoid arthritis. IDrugs. 1998;1(4):429-41.

35. Stroncek JD, Reichert WM. Indwelling neural implants: strategies for contending with the in vivo environment. Boca Raton: CRC Press; 2008.

36. Bodner L, Kaffe I, Littner MM, Cohen J. Extraction site healing in rats. A radiologic densitometric study. Oral Surg Oral Med Oral Pathol. 1993;75(3):367-72. doi:10.1016/0030-4220(93)90153-U

37. Feher A, Koivunemi A, Koivunemi M, Fuchs RK, Burr DB, Phipps RJ et al. Bisphosphonates do not inhibit periosteal bone formation in estrogen deficient animals and allow enhanced bone modeling in response to mechanical loading. Bone. 2010;46(1):203-7. doi:10.1016/j.bone.2009.10.023 